\title{
New Synthesis: The Chemistry of Partner Choice in Insect-Microbe Mutualisms
}

\author{
Peter H. W. Biedermann • Martin Kaltenpoth
}

Published online: 5 February 2014

(C) Springer Science+Business Media New York 2014

\begin{abstract}
Most insects are associated with mutualistic microorganisms that can confer novel traits and thereby play important roles for the ecology and evolution of host organisms. Although many insects ensure that their offspring are endowed with the beneficial symbionts by transmitting them vertically from parent to offspring, others rely on environmental uptake in every generation. How can these insects choose the best cooperative partner from the diversity of environmental microbes? Proposed means of partner choice - signaling and screening (Archetti et al. 2011) typically are chemically-mediated in cross-kingdom mutualisms. While wellstudied in plant-pollinator and some plant-microbe mutualisms, however, the use of chemical signals (e.g., specific odors, surface attractants) or selective environments (e.g., through nutritional constraints or defensive chemicals) to filter potential partners remains largely unexplored in insect-microbe associations, despite the implications for the ecology and evolution of the host organisms.
\end{abstract}

Ideally, individuals identify beneficial partners in advance of any interaction, thus preventing exploitation of the costly cooperative act. Signaling is a wellknown mechanism for partner selection in animal-animal (e.g., cleaner fish), animal-plant (e.g., pollinator and seed dispersal mutualisms), as well as plantmicrobe mutualisms (e.g., legumes and rhizobia, plants and mycorrhizal fungi). Signal honesty is required in order to maintain a mutualism, and thus signals most easily evolve if directly linked to partner quality. In insects, specific long-range signaling and short-distance recognition of mutualistic partners by odors or surface chemicals has been implicated in the establishment of symbiosis in several fungusgrowing insects. Some ambrosia beetle species are attracted to their fungal symbionts, suggesting that signaling by specific microbial volatile organic compounds (MVOCs) mediates the initial stages of partner choice (Hulcr et al. 2011). Long-range beetle attraction by general MVOCs (e.g., short-chain alcohols) is long known, but partner recognition is expected to involve specific secondary compounds (e.g., terpenoids) or blends of general MVOCs. However, since ambrosia beetles usually transmit their symbiotic fungus vertically in specialized pouches (mycangia) and inoculate them into new host trees upon colonization, attraction to the symbiont's volatiles may direct the beetles to suitable environments for fungus growth rather than providing a signal necessary for symbiosis establishment. By contrast, most fungus-growing termites collect their cultivar's spores de novo from the environment in each generation, highlighting the need for mechanisms to localize and recognize the symbiotic partner. Currently, it is unknown whether recognition occurs through volatile signals or surface compounds produced by the fungal spores. Given that spores often are rich in defensive secondary metabolites (e.g., terpenoids, mycotoxins), derivatives of these compounds could be involved in partner choice. Similar to ambrosia beetles and despite vertical transmission of their symbionts, leaf-cutter ants of the genus Acromyrmex chemically recognize their native cultivar fungi (see references in Zhang et al. 2007), as well as discriminate between native and non-native

P. H. W. Biedermann · M. Kaltenpoth $(\bowtie)$

Insect Symbiosis Research Group, Max Planck Institute for Chemical Ecology, Hans-Knoell-Str. 8, 07745 Jena, Germany

e-mail: mkaltenpoth@ice.mpg.de protective bacterial Pseudonocardia symbionts (Zhang et al. 2007), thus allowing for partner choice in a tripartite symbiosis. Although the mechanism for recognition of the bacteria remains unknown, the symbiont's chemical profile has been suggested as the most probable signal.

As an alternative to signaling, a host can provide a selective environment that excludes all but the highest quality partners through chemically-mediated screening (Archetti et al. 2011). For example, Euprymna scolopes squids create an environment in which bacterial bioluminescence protects the symbionts against the host's lethal reactive oxygen species (ROS), thus allowing only bioluminescent Vibrio fisheri bacteria to colonize the light organ (see references in Archetti et al. 2011). In insects, screening could play a role in associations where symbionts are seeded onto a substrate to serve as a nutritional resource (e.g., fungus-farming insects). For example, wood-boring bark and ambrosia beetles may screen their bacterial and fungal (food-) symbionts indirectly by choosing selective tree substrates, i.e., varying in nutrient and/or terpenoid composition. Defensive symbioses with Actinobacteria, such as those found in leaf-cutter ants and beewolf digger wasps, are another setting in which screening mechanisms can be expected. Hosts may indirectly select for high-quality defensive symbionts by providing abundant resources and thereby stimulating competition among potential candidates, with the - likely well-defended - superior competitor finally winning out. Thus, both primary and secondary host metabolites can be important for screening-based partner choice, by defining the symbionts' nutritional and competitive environment, respectively. Noteworthy - although their implications for partner choice and the evolution of mutualisms remain to be elucidated - the chemical nature and primary functions of many of these compounds may already be known.

Partner choice is a central aspect of the origin and evolutionary maintenance of mutualistic associations. As more and more taxonomically and functionally diverse symbioses between insects and microorganisms are discovered, understanding the mechanistic and molecular basis of signaling and screening to ensure partner specificity becomes increasingly important. Since chemical interactions between the symbiotic partners likely mediate partner choice in the majority of associations, contributions from the discipline of chemical ecology will greatly enhance our knowledge about the ecological and evolutionary processes governing specificity in mutualistic interactions.

\section{References}

Archetti M, Scheuring I, Hoffman M, Frederickson ME, Pierce NE, Yu DW (2011) Economic game theory for mutualism and cooperation. Ecology Lett 14:1300-1312

Hulcr J, Mann R, Stelinski LL (2011) The scent of a partner: ambrosia beetles are attracted to volatiles from their fungal symbionts. J Chem Ecol 37 : 1374-1377

Zhang MM, Poulsen M, Currie CR (2007) Symbiont recognition of mutualistic bacteria by Acromyrmex leaf-cutting ants. ISME J 1(4):313-320 